\title{
Cysteine degradation gene yhaM, encoding cysteine desulfidase, serves as a genetic engineering target to improve cysteine production in Escherichia coli
}

\author{
Gen Nonaka* (1) and Kazuhiro Takumi
}

\begin{abstract}
Cysteine is an important amino acid for various industries; however, there is no efficient microbial fermentation-based production method available. Owing to its cytotoxicity, bacterial intracellular levels of cysteine are stringently controlled via several modes of regulation, including cysteine degradation by cysteine desulfhydrases and cysteine desulfidases. In Escherichia coli, several metabolic enzymes are known to exhibit cysteine degradative activities, however, their specificity and physiological significance for cysteine detoxification via degradation are unclear. Relaxing the strict regulation of cysteine is crucial for its overproduction; therefore, identifying and modulating the major degradative activity could facilitate the genetic engineering of a cysteine-producing strain. In the present study, we used genetic screening to identify genes that confer cysteine resistance in E. coli and we identified yhaM, which encodes cysteine desulfidase and decomposes cysteine into hydrogen sulfide, pyruvate, and ammonium. Phenotypic characterization of a yhaM mutant via growth under toxic concentrations of cysteine followed by transcriptional analysis of its response to cysteine showed that yhaM is cysteine-inducible, and its physiological role is associated with resisting the deleterious effects of cysteine in E. coli. In addition, we confirmed the effects of this gene on the fermentative production of cysteine using E. coli-based cysteine-producing strains. We propose that yhaM encodes the major cysteine-degrading enzyme and it has the most significant role in cysteine detoxification among the numerous enzymes reported in E. coli, thereby providing a core target for genetic engineering to improve cysteine production in this bacterium.
\end{abstract}

Keywords: Cysteine degradation, Cysteine desulfidase, Cysteine desulfhydrase, Cysteine fermentation, yhaM

\section{Introduction}

Cysteine is an important amino acid for various industries, including pharmaceutical, food, and cosmetics. Bacterial fermentation is the major production method for many amino acids because it combines cost effectiveness and scalability. However, cysteine is one of the few amino acids for which efficient fermentation-based methods are not available, which is a major challenge for the amino acid fermentation industry (Wada and Takagi

\footnotetext{
*Correspondence: gen_nonaka@ajinomoto.com

Research Institute for Bioscience Products and Fine Chemicals, Ajinomoto Co., Inc., 1-1 Suzuki-cho, Kawasaki-ku, Kawasaki, Kanagawa 210-8681, Japan
}

2006). Cysteine is known to be highly toxic to cells (Hennicke et al. 2013; Sorensen and Pedersen 1991); therefore, cells are equipped with multifaceted and complex regulatory systems to manage its deleterious effects, thus facilitating strict control of the intracellular cysteine levels (Kredich 2008). Stringent metabolic regulatory control and toxicity of cysteine are the main factors that hinder the establishment of an effective production method in bacteria. Understanding the complexity of this regulatory process is crucial for the optimization of metabolic flux toward cysteine to facilitate its over-production.

The mechanisms responsible for regulating cysteine synthesis include feedback inhibition of key metabolic enzymes in the cysteine biosynthetic pathway, i.e., serine 
acetyltransferase (SAT) and 3-phosphoglycerate dehydrogenase (3-PGDH). These enzymes play major roles in controlling cysteine biosynthesis through feedback inhibition by cysteine and serine, respectively (Al-Rabiee et al. 1996; Kai et al. 2006). A master regulator of sulfur metabolism, CysB, controls the expression of most of the transporter genes involved in the uptake of various sulfur-containing compounds across cell membranes, as well as that of biosynthetic genes responsible for incorporating these sulfur sources into cysteine (Jagura-Burdzy and Kredich 1983; Kredich 1992; Yamazaki et al. 2016). The CysB-mediated induction of sulfur-assimilating genes promotes cysteine synthesis by coordinating carbon and sulfur flow when cysteine is in short supply. The efflux of excess cysteine using a specific cysteine exporter is another mode of regulation. The cysteine-inducible efflux pump encoded by cefA in Pantoea ananatis is known to be involved in this regulation by serving as a safety valve when cysteine is present in excess (Takumi and Nonaka 2016).

The degradation of cysteine by specific enzymes comprises an additional mode of regulation. Cysteine desulfhydrases (CDs) are among the best-characterized major cysteine-degrading enzymes in Escherichia coli and related bacteria (Awano et al. 2003, 2005; Takumi and Nonaka 2016). In addition, cysteine desulfidases are alternative cysteine-degrading enzymes that are less well-characterized as mediators of cysteine regulation compared to CDs (Tchong et al. 2005). Both enzymes catalyze the same reaction where cysteine is decomposed into sulfide, ammonia, and pyruvate. While cysteine desulfidases use a $[4 \mathrm{Fe}-4 \mathrm{~S}]$ center, $\mathrm{CDs}$ use a pyridoxal phosphate center to catalyze the hydrolysis of cysteine to sulfide. In some bacteria, a single major CD plays a central role in cysteine resistance through degradation, including CcdA in P. ananatis and its Salmonella enterica serovar Typhimurium ortholog CdsH (Oguri et al. 2012; Takumi and Nonaka 2016). These enzymes are cysteine-inducible and they are mediated by a specific transcription regulator, i.e., CcdR in $P$. ananatis and its $S$. enterica ortholog DecR; therefore, it has been proposed that they are involved in a specific mechanism for coping with cysteine toxicity (Oguri et al. 2012; Takumi and Nonaka 2016). In contrast, at least five minor enzymes (CysK, CysM, MetC, TnaA, and MalY) exhibit CD activities in E. coli and it has been proposed that they remove excess cysteine (Awano et al. 2005). However, the functional significance of these enzymes in cysteine degradation is controversial because their regulation associated with cysteine is insignificant (Nonaka, unpublished data; see "Discussion"); moreover, they mediate other primary physiological functions via distinctive enzymatic reactions [e.g., CysK and CysM are cysteine synthases; (Becker et al. 1969; Zhao et al. 2006)].

Escherichia coli and P. ananatis have been extensively studied as hosts for the fermentative production of cysteine (Takumi et al. 2017; Wada and Takagi 2006). Deregulation of the key enzymes related to feedback inhibition is the usual method employed for bacterial amino acid production. Deregulating the two key feedback loops by introducing feedback inhibition-insensitive mutants of 3-PGDH and SAT are the main options for cysteine overproduction (Al-Rabiee et al. 1996; Kai et al. 2006). Enhancing cysteine efflux pumps is also crucial for the downregulation of intracellular cysteine synthesis to bypass any toxic effects as well as for the secretion of cysteine into the fermentation medium to facilitate further isolation processes. Several efflux pumps are known to facilitate cysteine production including YdeD, YfiK, and multidrug exporters in E. coli; and CefA and CefB in P. ananatis (Dassler et al. 2000; Franke et al. 2003; Takumi and Nonaka 2016; Takumi et al. 2017; Yamada et al. 2006). Management of intracellular degradation activity is also essential for improving production; therefore, CDs and many other degradative enzymes have been identified and studied extensively. In $P$. ananatis, the discovery of $c c d A$, the only major $\mathrm{CD}$ encoded by this bacterium, almost fully addressed the problem because deletion of this gene virtually eliminated the cellular degradation of cysteine, thereby drastically increasing its synthesis (Takumi et al. 2017). Similarly, the deletion of CDs in E. coli was shown to be effective for increasing the synthesis of cysteine, where the stepwise deletion of known CDs decreased the intracellular CD activity, resulting in increased cysteine synthesis proportional to the number of deleted genes (Awano et al. 2005). However, even after the complete deletion of the five known CDs in E. coli, significant hydrogen sulfide-forming cysteine degradation activity levels were detected, which suggests that additional CDs or cysteine desulfidases that presumably have negative effects on cysteine synthesis may need to be identified. Moreover, a cysteine-inducible cysteine degradative activity was observed in the quintuple mutant, which suggests that at least one of the unknown decomposers of cysteine that remain to be identified might be cysteine-responsive (Awano et al. 2005). Identifying the unknown gene that possibly encodes a core cysteine degradation enzyme in $E$. coli will substantially contribute to our physiological understanding of cysteine metabolism and will thus help overcome the challenge of optimizing the fermentative production of cysteine in E. coli. 


\section{Materials and methods}

\section{Strains, plasmids, and growth conditions}

Bacterial strains and plasmids used in the present study are listed in Table 1 . All of the strains were grown at $37{ }^{\circ} \mathrm{C}$ in Luria-Bertani (LB) broth, M9 minimal medium (Sambrook and Russell 2001), or fermentation medium $\left(15 \mathrm{~g}\right.$ of $\left(\mathrm{NH}_{4}\right)_{2} \mathrm{SO}_{4}, 1.5 \mathrm{~g}$ of $\mathrm{KH}_{2} \mathrm{PO}_{4}, 1 \mathrm{~g}$ of $\mathrm{MgSO}_{4} \cdot 7 \mathrm{H}_{2} \mathrm{O}, 0.1 \mathrm{mg}$ of thiamine hydrochloride, $1.7 \mathrm{mg}$ of $\mathrm{FeSO}_{4} \cdot 7 \mathrm{H}_{2} \mathrm{O}, 0.15 \mathrm{mg}$ of $\mathrm{Na}_{2} \mathrm{MoO}_{4} \cdot 2 \mathrm{H}_{2} \mathrm{O}$, $0.7 \mathrm{mg}$ of $\mathrm{CoCl}_{2} \cdot 6 \mathrm{H}_{2} \mathrm{O}, 1.6 \mathrm{mg}$ of $\mathrm{MnCl}_{2} \cdot 4 \mathrm{H}_{2} \mathrm{O}, 0.3 \mathrm{mg}$ of $\mathrm{ZnSO}_{4} \cdot 7 \mathrm{H}_{2} \mathrm{O}, 0.25 \mathrm{mg}$ of $\mathrm{CuSO}_{4} \cdot 5 \mathrm{H}_{2} \mathrm{O}, 0.6 \mathrm{~g}$ of tryptone, $0.3 \mathrm{~g}$ of yeast extract, $0.6 \mathrm{~g}$ of $\mathrm{NaCl}, 20 \mathrm{~g}$ of $\mathrm{CaCO}_{3}$, $135 \mathrm{mg}$ of L-histidine $\mathrm{HCl} \cdot \mathrm{H}_{2} \mathrm{O}, 4 \mathrm{~g}$ of $\mathrm{Na}_{2} \mathrm{~S}_{2} \mathrm{O}_{3}, 2 \mathrm{mg}$ of pyridoxine hydrochloride, and $40 \mathrm{~g}$ of glucose per liter). The medium was supplemented with $20 \mu \mathrm{g} \mathrm{mL}{ }^{-1}$ kanamycin and/or $25 \mu \mathrm{g} \mathrm{mL}{ }^{-1}$ chloramphenicol as required.
Gene deletion was performed in $E$. coli using the $\lambda$-Red recombination system developed by Datsenko and Wanner (Datsenko and Wanner 2000). Plasmid pKD46, which carries an arabinose-inducible $\lambda$-Red gene, was used to facilitate Red recombination and $\mathrm{pMW}-\lambda a t t L-\mathrm{Km}^{\mathrm{R}}$ - $\lambda a t t R$ plasmids (Van Dien et al. 2010) were used as templates to obtain PCR-generated gene disruption cassettes for ::kan. The primers listed in Table 2 were used to generate these cassettes. To excise markers from the cassettes flanked by $a t t L / R$, we used the phage $\lambda$ site-specific Int/Xis system (Van Dien et al. 2010), where we employed the pMW-intxis plasmid encoding the Int/Xis recombinase.

To construct the $y h a M$ and $y d e D$ expression plasmids, multicopy cloning vectors pSTV29 (Takara, Tokyo, Japan) and pACYC177 (Nippon Gene, Tokyo, Japan) were used as backbones. The PCR-generated fragments of $y d e D$ and $y h a M$ were digested using BamHI (these

Table 1 Strains and plasmids

\begin{tabular}{|c|c|c|}
\hline Strains and plasmids & Description $^{\mathbf{a}}$ & Source or reference \\
\hline \multicolumn{3}{|l|}{ Strains } \\
\hline MG1655 & Wild-type E. coli MG1655 (ATCC 47076) & ATCC \\
\hline MG1655 $\Delta y h a M$ & MG1655 $\Delta y h a M$ & This study \\
\hline \multicolumn{3}{|l|}{ Plasmids } \\
\hline pSTV29 & Cloning vector, p15a ori, $\mathrm{Cm}^{r}$ & Takara \\
\hline pSTV-ydeD5 & pSTV29; ydeD (E. coli) gene containing upstream 300 bp and 200 bp downstream & This study \\
\hline pSTV-yhaM7 & pSTV29; yhaM (E. coli) gene containing upstream 300 and 200 bp downstream & This study \\
\hline pACYC177 & Cloning vector, p15a ori, $\mathrm{Km}^{r}$ & Nippon Gene \\
\hline pACYC-yhaM1 & pACYC177; yhaM (E. coli) gene containing upstream 300 and 200 bp downstream & This study \\
\hline pMIV-cysE5 & cysE5 (E. coli) under the control of the ompC promoter, p15a ori, $\mathrm{Cm}^{r}$ & (Nonaka and Takumi 2011) \\
\hline
\end{tabular}

ATCC American Type Culture Collection

a $\mathrm{Cm}^{\mathrm{r}}, \mathrm{Km}^{\mathrm{r}}$, resistance to chloramphenicol and kanamycin

Table 2 Oligonucleotides

\begin{tabular}{|c|c|c|}
\hline Primer name & Sequence $\left(5^{\prime}-3^{\prime}\right)$ & Use \\
\hline DyhaM-FW & $\begin{array}{l}\text { ATGTTTGATTCGACTTTAAATCCGTTATGGCAGCGTTACATCCTCGCCGTTGAAGCCTGCTTTTTTATACTAA- } \\
\text { GTTGGCA }\end{array}$ & Construction of $\triangle y h a M$ \\
\hline DyhaM-RV & $\begin{array}{l}\text { TTATCTGGCCTTGCTCGCCATAATCTCGATAATCTGCCGATCCGTTTGCTCGCTCAAGTTAGTATAAAAAA- } \\
\text { GCTGAACGA }\end{array}$ & Construction of $\triangle y h a M$ \\
\hline ECOydeD-F & CGCGGATCCAATGGTCATAAATGGCAGCGTAGCGC & Cloning of ydeD \\
\hline ECOydeD-R & CGCGGATCCGCAGGGCGTTGCGGAACAAAC & Cloning of ydeD \\
\hline ECOyhaM-F & CGCGGATCCAAGATGCCTGCCGAGAAGATTAACG & Cloning of yham \\
\hline ECOyhaM-R & CGCGGATCCGAGCGAGCTGGAAGCTATCG & Cloning of yham \\
\hline yhaM(Ec)RT-F & CTCGATTCCGCGAAGCTAAA & Real-time PCR: detection of yhaM \\
\hline yhaM(Ec)RT-R & CCCCCACTTACCGCTCAA & Real-time PCR: detection of yhaM \\
\hline yhaO(Ec)RT-F & GCCATTATTACGCTGCCGTTT & Real-time PCR: detection of yhaO \\
\hline yhaO(Ec)RT-R & CCATCGGACTTAACGTCTGGAT & Real-time PCR: detection of yhaO \\
\hline $\operatorname{metC}(\mathrm{Ec}) \mathrm{RT}-\mathrm{F}$ & AAGCCGCCACCAAATATCTG & Real-time PCR: detection of metC \\
\hline $\operatorname{metC}(\mathrm{Ec}) \mathrm{RT}-\mathrm{R}$ & ACACGGCAGTGCCAATCA & Real-time PCR: detection of metC \\
\hline
\end{tabular}


sites were designed based on the $5^{\prime}$ and $3^{\prime}$ ends of the primers) and cloned into the corresponding sites in pSTV29 to yield pSTV-ydeD5 and pSTV-yhaM7, and in pACYC177 to yield pACYC-yhaM1. The primers used for plasmid construction are listed in Table 2.

\section{Screening for genes that confer cysteine resistance}

Genomic DNA from E. coli wild-type strain MG1655 was digested partially with Sau3AI and fragments measuring approximately $10 \mathrm{~kb}$ were cloned into the BamHI site of pSTV29 (Takara Bio, Shiga, Japan). The genomic library obtained was introduced into MG1655 by electroporation and the cells were then challenged on M9 plates containing 2-4 mM cysteine for 2 days. Colonies were isolated and candidate genes in the plasmids that conferred cysteine resistance were analyzed by sequencing.

\section{Growth assay}

For the growth assay on plates, overnight cultures produced on regular M9 minimal medium plates (containing $\mathrm{MgSO}_{4}$ as a sulfur source) were streaked onto fresh M9 sulfur-free plates $\left(\mathrm{MgSO}_{4}\right.$ was replaced with $\left.\mathrm{MgCl}_{2}\right)$ supplemented with $4 \mathrm{mM}$ cysteine and then incubated at $37^{\circ} \mathrm{C}$ for 2 days before observing their growth.

For the growth assay in liquid culture, each overnight culture cultivated in a test tube containing $3 \mathrm{~mL}$ of $\mathrm{M} 9$ minimal medium was diluted 1:100 with fresh M9 minimal medium to obtain a total volume of $3 \mathrm{~mL}$, before growing overnight in test tubes with agitation. The cells were then inoculated into $4 \mathrm{~mL}$ of fresh $\mathrm{M} 9$ minimal medium in test tubes containing 0 or $100 \mu \mathrm{M}$ of cysteine to obtain an initial optical density at $600 \mathrm{~nm}\left(\mathrm{OD}_{600}\right)$ of 0.005. Growth $\left(\mathrm{OD}_{660}\right)$ was monitored automatically in a TN-1506 incubator (Advantec Toyo, Tokyo, Japan).

\section{Quantitative RT-PCR}

A 1:100 dilution of an overnight culture of $E$. coli MG1655 grown in M9 minimal medium was inoculated into $25 \mathrm{~mL}$ of the same fresh M9 medium in a flask. Cysteine $(100 \mu \mathrm{M})$ was added after cells reached logarithmic phase (approximately $7 \mathrm{~h}$ ) with $\mathrm{OD}_{600} \sim 0.3$. Samples were obtained after 0,5 , and $20 \mathrm{~min}$ and they were mixed with RNA Protect Bacteria Reagent (Qiagen, USA), frozen in liquid $\mathrm{N}_{2}$, and stored at $-80{ }^{\circ} \mathrm{C}$. Total RNA was prepared using an RNeasy Mini Kit (Qiagen, USA). Subsequently, the samples were treated with DNase TURBO DNA-free (Ambion, USA), followed by reverse transcription using an ExScript RT Reagent Kit (Takara Bio, Japan). Using the primers listed in Table 2 and a Power SYBR Green Master Mix (Applied Biosystems, USA), cDNA was quantified by real-time PCR according to the manufacturer's instructions. Transcript levels were normalized against that of the $16 \mathrm{~S}$ ribosomal RNA internal standard.

\section{Fermentative production of cysteine}

Each strain was streaked onto an LB plate and grown overnight. Cells were collected using a $10-\mu \mathrm{L}$ loop, which was passed through $7 \mathrm{~cm}$ of bacteria spread, were fully grown on the culture plates, and then inoculated into $2 \mathrm{~mL}$ of fermentation medium in test tubes $(23 \mathrm{~mm}$ internal diameter $\times 200 \mathrm{~mm}$ length). The cells were incubated at $32{ }^{\circ} \mathrm{C}$ with agitation until all sugar was consumed, unless stated otherwise. The cysteine level in the culture was quantitatively analyzed according to the method described by Gaitonde (Gaitonde 1967). Before adding the ninhydrin reagent, samples were reduced by incubation with $10 \mathrm{mM}$ dithioerythritol in $10 \mathrm{mM}$ Tris $-\mathrm{HCl}$ buffer at $\mathrm{pH} 8.5$ for $10 \mathrm{~min}$. This assay system facilitates the determination of cysteine in its oxidized form (cystine) as well as its condensation product with pyruvate (2-methyl-2,4-thiazolidine carboxylic acid) (Dassler et al. 2000; Wiriyathanawudhiwong et al. 2009). Since these derivatives are easily converted to cysteine via either simple biological or chemical reaction, the assay provides an informative output for the evaluation of ability to produce cysteine as the total cysteine. Cysteine yield was defined as grams of the final amount of the product obtained from $1 \mathrm{~g}$ of glucose, which was expressed as a percentage.

\section{Results}

Screening the genomic library for genes that confer cysteine resistance identified genes involved in cysteine efflux and cysteine degradation

To identify $E$. coli genes that confer cysteine resistance when overexpressed, wild-type bacterial strain MG1655 was transformed with a multicopy genomic library of MG1655 and cells were challenged on M9 minimal medium plates supplemented with 2-4 $\mathrm{mM}$ cysteine. These conditions showed that the toxicity of cysteine was sufficient to inhibit colony formation for up to 2 days. Colonies were selected after 2 days and the genes that contributed to this phenotype were identified by sequencing the plasmid DNA. In our previous studies, we screened a genomic library of $P$. ananatis using the same system and obtained genes involved in cysteine efflux (cefA and $c e f B$ ) and degradation $(c c d A)$ (Takumi and Nonaka 2016). The genes obtained were validated as targets for metabolic engineering for cysteine fermentation based on $P$. ananatis either by enhancing the efflux or decreasing the degradation activity, and they were shown to be essential for establishing the overproduction of cysteine (Takumi et al. 2017). Therefore, this screening system is suitable for identifying significant factors. 
In the present study, screening using $E$. coli identified two loci containing $y d e D$, a cysteine efflux pump involved in the cysteine/cystine shuttle system that protects cells from reactive oxygen species (Dassler et al. 2000; Ohtsu et al. 2010) and yhaM, a homolog of the Methanocaldococcus jannaschii cdsB, which encodes a cysteine desulfidase that is presumably involved in cysteine degradation (Tchong et al. 2005). Screening conditions were reproduced using $y d e D$ and $y h a M$ cloned from $E$. coli, each containing $300 \mathrm{bp}$ upstream and $200 \mathrm{bp}$ downstream of the corresponding genes, thereby confirming that the resistance to cysteine was related to these genes (Fig. 1). Figure 2 shows the growth of E. coli wild-type strain MG1655 when challenged under conditions containing toxic levels of cysteine $(100 \mu \mathrm{M})$ in M9 minimal medium. Overexpression of yhaM improved growth in these conditions, which was consistent with the typical effects of enhanced cysteine degradation (the lag reduced; see (Takumi and Nonaka 2016) for the growth curve with P. ananatis $c c d A$ enhancement). Deletion of $y$ haM had negative effects on growth in the presence of cysteine, thereby suggesting that $\mathrm{YhaM}$ is involved in cysteine resistance via degradation. This screening system and phenotypic characterization of growth in the presence of cysteine identified factors related to efflux and degradation, the regulation of which may be effective for cysteine production (Takumi and Nonaka 2016). Indeed, one of the genes identified by screening, $y d e D$, has been studied and characterized extensively as a cysteine efflux pump with a central role in cysteine production

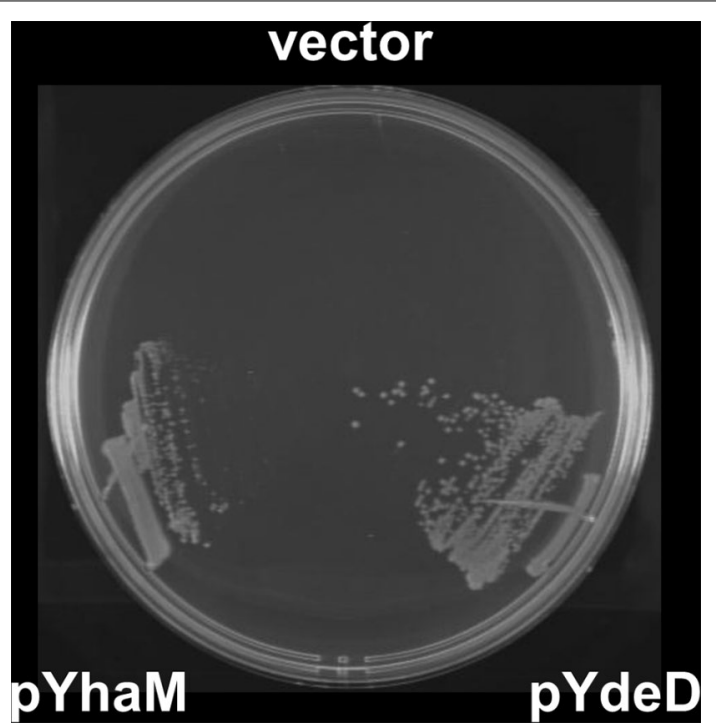

Fig. 1 YhaM confers cysteine resistance. E. coli MG1655 carrying vector (pSTV29), ydeD on plasmid (pSTV-ydeD5), and yhaM on plasmid (pSTV-yhaM7) were streaked on a M9 plate containing $4 \mathrm{mM}$ cysteine and incubated at $37^{\circ} \mathrm{C}$ for 2 days

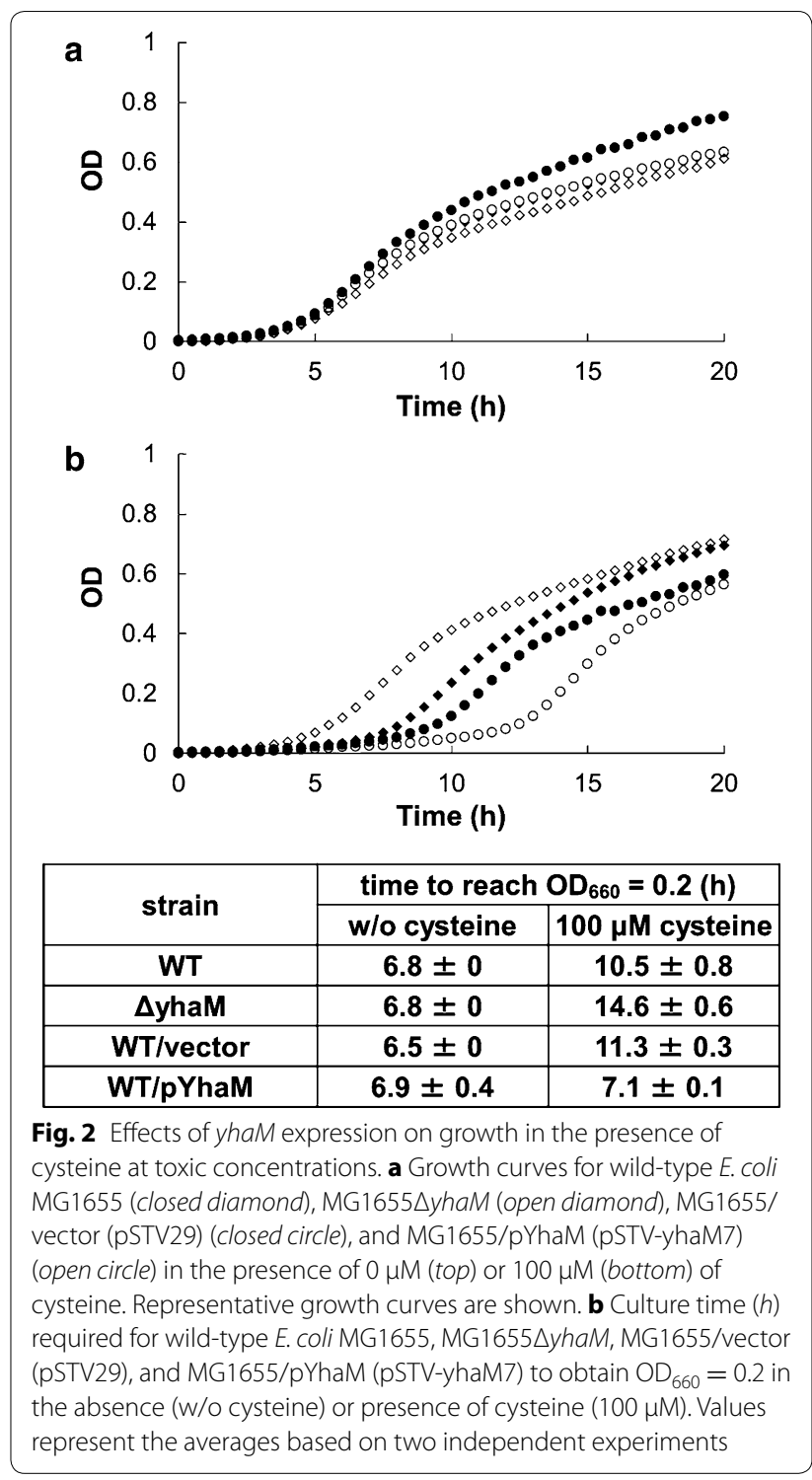

by $E$. coli (Dassler et al. 2000). Therefore, we focused on yhaM to characterize its possible application in cysteine production.

\section{yhaM is cysteine inducible}

To explore the physiological significance of YhaM for the detoxification of cysteine in E. coli, we performed transcriptional analysis to probe its expression in response to cysteine shock (addition of exogenous cysteine) using quantitative RT-PCR. Figure 3 shows the mRNA levels of $y h a O$ and $y h a M$, with metC as a negative control. $y h a O$ is located upstream of $y h a M$ and they are predicted to be organized in an operon (regulon DB; http://regulondb. ccg.unam.mx/). Our findings demonstrated that $y$ haM and $y h a O$ expression was significantly induced after cysteine shock. The response was rapid (less than $5 \mathrm{~min}$ ) 


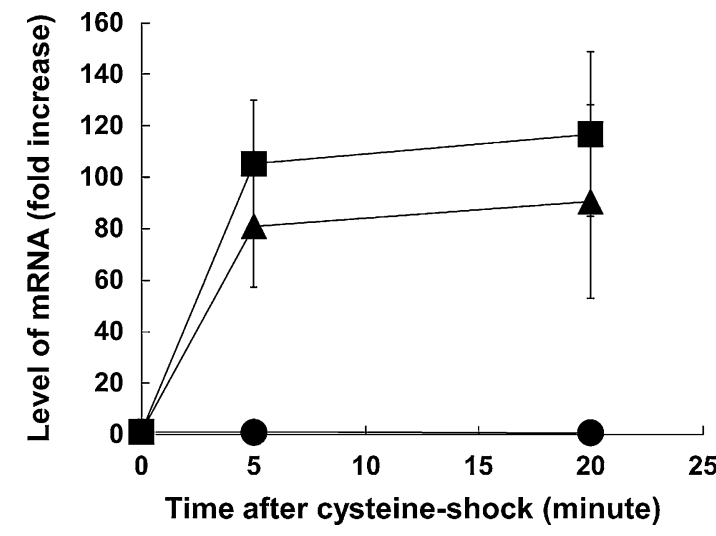

Fig. 3 Induction of yhaO and yhaM transcription by cysteine. Time course of the relative yhaO (square), yhaM (triangle), and metC (circle) mRNA levels after the addition of cysteine (cysteine-shock). Relative mRNA levels compared with the mRNA level before cysteine addition are shown. Values represent the averages of the results based on three independent experiments and the error bar represents one standard deviation

and strong (ca 80- and 100-fold increases for yhaM and $y h a O$, respectively), which is consistent with an idea that YhaM is responsive to cysteine (see "Discussion" for the significance of the speed and magnitude of the induction). The results support the hypothesis that YhaM acts in cysteine degradation, which is associated with cysteine detoxification in E. coli [see "Discussion" for the function of YhaM deduced from this study as well as previous studies (Shimada et al. 2016; Takumi et al. 2017; Tchong et al. 2005)].

\section{YhaM modulates cysteine production}

YhaM serves as a primary degradation enzyme for cysteine detoxification via cysteine-mediated induction in E. coli, so it may affect cysteine production and it could be an important target for the genetic engineering of cysteine-producing strains of $E$. coli. To explore this possibility, we deleted and overexpressed the yhaM gene in a model cysteine-producing strain based on $E$. coli MG1655 and performed a fermentation test to evaluate its production of cysteine. In E. coli, cysteine synthesis is regulated primarily by SAT, which is encoded by $c y s E$, and it is subject to feedback inhibition by cysteine. Cells that express a feedback-insensitive mutant of SAT produce traces of cysteine and they can be exploited as a simple cysteine-producing strain (Yamada et al. 2006). Efflux of the synthesized cysteine from the cells is the production bottleneck for this model strain, where only traces of the cysteine produced accumulate in the production medium and most of the product is decomposed in the cells by degradation enzymes. Indeed, the production of cysteine in this model strain based on P. ananatis (i.e., $P$. ananatis carrying the mutant SAT), a close relative and an alternative promising host to $E$. coli, was increased by deleting the major $\mathrm{CD}, \mathrm{CcdA}$ (Takumi et al. 2017). Therefore, E. coli carrying the mutant SAT is a suitable model for evaluating the effects of YhaM. Therefore, we employed the E. coli wild-type strain MG1655 harboring the plasmid pMIV-cysE5 carrying a cysE5 gene (Kai et al. 2006), which encodes a feedback-insensitive mutant of SAT, to determine whether yhaM expression can affect cysteine production. Tables 3 and 4 show the fermentative production of cysteine under conditions where $y h a M$ was overexpressed from a multicopy plasmid and where it was deleted, respectively. Production of cysteine decreased significantly when $y h a M$ was overexpressed (Table 3) whereas it increased drastically when yhaM was deleted (Table 4), which suggests that YhaM was in an active state during cysteine production when it was present. These findings suggest that $y h a M$ may be a promising target for genetic engineering to obtain highperformance cysteine-producing strains at industrial production levels.

\section{Discussion}

YhaM was originally characterized as a cysteine desulfidase in M. jannaschii (Tchong et al. 2005). Recently, Ishihama et al. characterized YhaM in E. coli, where they demonstrated that $y h a M$ encodes a cysteine-inducible hydrogen sulfide-forming cysteine degradative enzyme, which is involved in the detoxification of cysteine (Shimada et al. 2016). These findings are consistent with our claim that YhaM may catalyze the main degradative activity of cysteine in E. coli, thus making it one of the key factors required for establishing efficient fermentative production of cysteine. Our screening study focused on cysteine resistance, characterization of a direct cysteine stimulon with a rapid and strong response, and its applications to cysteine fermentation, where these approaches

Table 3 Effects of yhaM overexpression on the production of cysteine

\begin{tabular}{lllll}
\hline Strain & Plasmid & Cysteine $\left(\mathbf{m g ~ L}^{-\mathbf{1}}\right)$ & Yield (\%) & OD $_{\mathbf{6 6 0}}$ \\
\hline MG1655 (WT) & pMIV-cysE5, pACYC177 & $74.6 \pm 7.0$ & $0.187 \pm 0.018$ & $21.7 \pm 0.8$ \\
& pMIV-cysE5, pACYC-yhaM1 & $14.9 \pm 1.1$ & $0.037 \pm 0.003$ & $22.0 \pm 0.8$ \\
\hline
\end{tabular}

Values represent the averages based on five independent experiments and the error value represents one standard deviation. To retain the plasmids, $20 \mu \mathrm{g} \mathrm{L}^{-1}$ kanamycin and $25 \mathrm{~g} \mathrm{~mL}^{-1}$ chloramphenicol were added. Cultivation was terminated at $21-24 \mathrm{~h}$ after inoculation when all the sugar ( $40 \mathrm{~g} \mathrm{\textrm {L } ^ { - 1 }}$ ) had been consumed 
Table 4 Effects of yhaM deletion on the production of cysteine

\begin{tabular}{lccll}
\hline Strain & Plasmid & $\begin{array}{c}\text { Cysteine } \\
\left(\mathbf{m g ~ L}^{-\mathbf{1}} \mathbf{)}\right.\end{array}$ & Yield (\%) & OD $_{\mathbf{6 6 0}}$ \\
\hline MG1655 (WT) & pMIV-cysE5 & $64.6 \pm 4.5$ & $0.164 \pm 0.011$ & $28.6 \pm 0.8$ \\
$\begin{array}{l}\text { MG1655 } \\
\text { DyhaM }\end{array}$ & pMIV-cysE5 & $316.2 \pm 74.4$ & $0.810 \pm 0.195$ & $29.4 \pm 0.9$ \\
& & & & \\
\hline
\end{tabular}

Values represent the averages based on four independent experiments and the error value represents one standard deviation. To retain the plasmids, $25 \mu \mathrm{g} \mathrm{mL} \mathrm{m}^{-1}$ chloramphenicol was added. Cultivation was terminated at $15-17 \mathrm{~h}$ after inoculation when $>95 \%$ of the total sugar $\left(>38 \mathrm{~g} \mathrm{~L}^{-1}\right)$ had been consumed

provided important insights into the crucial factors that affect cysteine fermentation using $E$. coli.

Multiple enzymes have $\mathrm{CD}$ activities in E. coli, but most of them are involved in other physiological functions via the distinct enzymatic activity of $\mathrm{CD}$, so the significance of their activity and roles in cysteine degradation remain unclear. Based on our findings as well as findings from recent studies, we propose that $y h a M$ encodes the major cysteine degradation enzyme in E. coli. Several key findings support this hypothesis. First, our screening approach is well established where it identified the major CD called CcdA and its cysteine-responsive regulator CcdR in $P$. ananatis (Takumi and Nonaka 2016). It is important to note that none of the known CDs in $E$. coli were identified by screening, which suggests that their contributions as cysteine scavengers might be insignificant. Second, yhaM expression was cysteineinducible. In $E$. coli, tnaA was reported to be cysteineresponsive (Awano et al. 2005), but our cysteine-shock experiment failed to detect cysteine-mediated induction (data not shown), which indicates that tnaA is not a direct cysteine stimulon. Findings from a recent study are consistent with our finding concerning the cysteinemediated induction of $y h a M$, where the $y h a M$ mRNA levels were upregulated when cells were cultivated in the presence of cysteine (Shimada et al. 2016). The present study investigated the properties based on kinetics, where we showed that the induction of yhaM expression in response to cysteine was significant in terms of both its speed and magnitude, which strongly suggests that the induction was direct. Third, $y h a M$ is organized in an operon with $y h a O$, which encodes a cysteine transporter (Shimada et al. 2016). Severity of cysteine toxicity in the periplasm was recently demonstrated (Ohtsu et al. 2010, 2015). Therefore, it is reasonable that cells control the main decomposer as well as a specific transporter at the transcriptional level, which may explain why $y h a O$ has $y h a M$ as a partner among the many CDs. Finally, modulating the expression of $y \mathrm{haM}$ via deletion or overexpression drastically affected the ability of the cells to produce cysteine. These phenotypic features are consistent with our previous findings in $P$. ananatis based on its main CD encoded by $c c d A$ (Takumi and Nonaka 2016).

Recently, the induction of the YhaM by cysteine was shown to be mediated by a transcriptional regulator $\mathrm{YbaO}$ (Shimada et al. 2016). Interestingly, the major cysteine degradation enzyme in $P$. ananatis, CcdA (and its $\mathrm{S}$. enterica ortholog, $\mathrm{CdsH}$ ), is also cysteine-inducible, where induction is mediated by the $\mathrm{YbaO}$ ortholog of $P$. ananatis, CcdR (S. enterica ortholog, DecR) (Oguri et al. 2012; Takumi and Nonaka 2016). Therefore, these two bacterial cysteine degradation systems (i.e., YhaM and $\mathrm{CcdA} / \mathrm{CdsH}$ ) that catalyze the same cysteine-decomposing reaction, share the same transcriptional regulator (i.e., $\mathrm{YbaO} / \mathrm{CcdR} / \mathrm{DecR}$ ). However, they share poor sequence identity, and thus they are classified as different enzymes (CD for $\mathrm{CcdA} / \mathrm{CdsH}$ and cysteine desulfidase for YhaM). In P. ananatis (and S. enterica), $c c d A$ is located adjacent to $c c d R$ on the genome and they form a gene cluster. On the other hand, in E. coli, ccdA is missing from the position adjacent to $y b a O$ and the $y h a O M$ operon is instead located at another locus in the genome. These findings suggest an interrelationship between two distinct cysteine degradation systems via a common transcriptional regulator $\mathrm{CcdR} / \mathrm{DecR} / \mathrm{YbaO}$ among bacteria, which motivated us to explore the conservation and variation of these systems among bacteria (see Fig. 4a for a summary of the main components). YhaM-YhaO is conserved in a limited number of bacterial species. It is mainly conserved among some $\gamma$-Proteobacteria members, particularly Enterobacteriaceae. Interestingly, this conservation varies even within a single species, e.g., both $y h a O$ and $y h a M$ are present in E. coli K-12 and O157:H1, but only yhaM is present in CFT073. Likewise, S. enterica CT18 contains $y$ haOM, whereas LT2 contains only yhaM. Moreover, conservation of the CcdR-CcdA/ DecR-CdsH system is also limited to $\gamma$-Proteobacteria, particularly Enterobacteriaceae. We queried the STRING database (Snel et al. 2000) to obtain an overview of the conservation and variation in the arrangement of genes among the bacteria in Enterobacteriaceae and identified seven groups based on the genome context (Fig. 4b). Group-A and group-B comprise the $P$. ananatis-type and the $E$. coli-type gene arrangements, respectively. Interestingly, a group of bacteria carry the full set of genes, i.e., group-C. Group-D bacteria contain the regulator $y b a O / c c d R$, which is missing with its counterpart $c c d A$ and $y h a O M$. Thus, even in the same Enterobacteriaceae families, variations in the bacterial conservation of this system presumably reflect their complex requirements under specific environmental conditions, which have developed by evolution. Understanding the entire degradation systems and determining the necessary genetic modifications required for their targeting are crucial for 
a

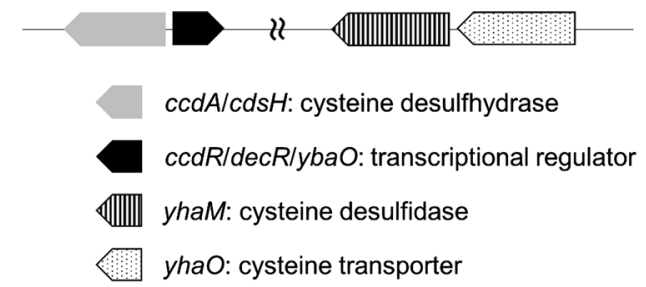

b

\begin{tabular}{|c|c|}
\hline Group-A & $\begin{array}{l}\text { Pantoea ananatis, Enterobacter sp. 638, Enterobacteriaceae bacterium, } \\
\text { Cronobacter sakazakii, Serratia proteamaculans, Rahnella aquatilis, } \\
\text { Pectobacterium atrosepticum, Dickeya dadantii, Erwinia billingiae, } \\
\text { Photorhabdus luminescens, Vibrio cholerae, Oceanimonas sp. GK1, } \\
\text { Xenorhabdus bovienii }\end{array}$ \\
\hline Group-B & $\begin{array}{l}\text { Escherichia coli, Escherichia albertii, Shigella flexneri, Proteus mirabilis, } \\
\text { Edwardsiella tarda, Edwardsiella ictaluri }\end{array}$ \\
\hline Group-C & $\begin{array}{l}\text { Salmonella enterica, Salmonella bongori, Enterobacter cloacae, } \\
\text { Citrobacter rodentium, Citrobacter koseri, Yersinia enterocolitica }\end{array}$ \\
\hline Group-D & Providencia stuartii \\
\hline
\end{tabular}

Fig. 4 Conservation and variation of cysteine degradation systems in Enterobacteriaceae bacteria. a Genomic context of the genes in the bacterial cysteine degradation systems conserved in bacteria. $\mathbf{b}$ Comparison of the genomic contexts of the bacterial cysteine degradation systems among genomes of Enterobacteriaceae bacteria. Representative examples of bacterial genomes are shown. Note that Xenorhabdus bovienii contains yhaO, and some Escherichia coli strains (e.g., CFT073) and Salmonella enterica strains (e.g., CT18) lack yhaO; thus, they represent subgroups. Data were obtained using the web resource STRING (http://www.string-db.org/)

improving the bacterial fermentation-based production of cysteine.

Owing to the toxicity of cysteine, cells are equipped with complex mechanisms for its degradative regulation. Therefore, managing cellular degradation activity is an essential approach for establishing efficient fermentative production of cysteine. Having clarified the core decomposers of cysteine in $E$. coli, the next step to facilitate the regulation of cysteine degradation will be fine-tuning each of the degradative genes in combination with the cellular efflux capacity. In $E$. coli, except for YhaM, all cysteine degradation enzymes have physiological functions other than cysteine decomposition; simply deleting the CD genes might perturb important physiological functions, thereby negatively affecting the process of fermentation. Therefore, carefully selecting the target genes and fine-tuning their expression levels will be important for genetic engineering. In particular, yhaM might be the main target among the many genes involved in cysteine degradation because it is specific to cysteine and it may have less negative impacts on various fermentation profiles. Indeed, according to our findings, the deletion of yhaM showed no negative effects on the OD, cultivation time, and other fermentation parameters.
Stepwise genetic engineering of cysteine-producing strains has been demonstrated in P. ananatis, where the core factors related to cysteine production were modulated in combination, including feedback-insensitive mutant alleles of SAT and 3-PGDH, efflux pumps, and the major CD (Takumi et al. 2017). Furthermore, ratelimiting steps in the cysteine biosynthetic pathway were enhanced in addition to these core factors. Most of the core factors are currently known following the identification of the major and minor CDs in E. coli. Therefore, the next challenge for $E$. coli-based cysteine production will be stepwise genetic engineering by targeting and fine-tuning the core factors in combination with the rate-limiting biosynthetic genes.

\section{Abbreviations}

SAT: serine acetyltransferase; 3-PGDH: 3-phosphoglycerate dehydrogenase; CD: cysteine desulfhydrase; LB: Luria-Bertani; OD: optical density; RT-PCR: real time polymerase chain reaction; WT: wild type.

\section{Authors' contributions}

Both authors designed and conducted the experiments and evaluated the results. GN drafted the manuscript. KT revised the manuscript. Both authors read and approved the final manuscript.

\section{Acknowledgements}

We thank Hisao Ito, Kazuo Nakanishi, Mikhail Ziyatdinov, and Mikhail Gusyatiner for many helpful discussions. 


\section{Competing interests}

The authors declare that they have no competing interests.

\section{Availability of data and materials}

All data are fully available without restriction.

\section{Consent for publication}

This study does not contain any individual person's data.

\section{Ethics approval and consent participate}

This article does not contain any studies with human participants or animals performed by any of the authors.

\section{Funding}

The present study was supported by Ajinomoto Co. Inc.

\section{Publisher's Note}

Springer Nature remains neutral with regard to jurisdictional claims in published maps and institutional affiliations.

Received: 13 April 2017 Accepted: 21 April 2017

Published online: 10 May 2017

\section{References}

Al-Rabiee R, Zhang Y, Grant GA (1996) The mechanism of velocity modulated allosteric regulation in D-3-phosphoglycerate dehydrogenase. Site-directed mutagenesis of effector binding site residues. J Biol Chem 271(38):23235-23238

Awano N, Wada M, Kohdoh A, Oikawa T, Takagi H, Nakamori S (2003) Effect of cysteine desulfhydrase gene disruption on L-cysteine overproduction in Escherichia coli. Appl Microbiol Biotechnol 62(2-3):239-243. doi:10.1007/ s00253-003-1262-2

Awano N, Wada M, Mori H, Nakamori S, Takagi H (2005) Identification and functional analysis of Escherichia coli cysteine desulfhydrases. Appl Environ Microbiol 71(7):4149-4152. doi:10.1128/aem.71.7.4149-4152.2005

Becker MA, Kredich NM, Tomkins GM (1969) The purification and characterization of $\mathrm{O}$-acetylserine sulfhydrylase-A from Salmonella typhimurium. J Biol Chem 244(9):2418-2427

Dassler T, Maier T, Winterhalter C, Bock A (2000) Identification of a major facilitator protein from Escherichia coli involved in efflux of metabolites of the cysteine pathway. Mol Microbiol 36(5):1101-1112

Datsenko KA, Wanner BL (2000) One-step inactivation of chromosomal genes in Escherichia coli K-12 using PCR products. Proc Natl Acad Sci USA 97(12):6640-6645. doi:10.1073/pnas.120163297

Franke I, Resch A, Dassler T, Maier T, Bock A (2003) YfiK from Escherichia coli promotes export of $\mathrm{O}$-acetylserine and cysteine. J Bacteriol 185(4):1161-1166

Gaitonde MK (1967) A spectrophotometric method for the direct determination of cysteine in the presence of other naturally occurring amino acids. Biochem J 104(2):627-633

Hennicke F, Grumbt M, Lermann U, Ueberschaar N, Palige K, Bottcher B, Jacobsen ID, Staib C, Morschhauser J, Monod M, Hube B, Hertweck C, Staib P (2013) Factors supporting cysteine tolerance and sulfite production in Candida albicans. Eukaryot Cell 12(4):604-613. doi:10.1128/ec.00336-12

Jagura-Burdzy G, Kredich NM (1983) Cloning and physical mapping of the cysB region of Salmonella typhimurium. J Bacteriol 155(2):578-585

Kai Y, Kashiwagi T, Ishikawa K, Ziyatdinov MK, Redkina El, Kiriukhin MY, Gusyatiner MM, Kobayashi S, Takagi H, Suzuki E (2006) Engineering of Escherichia coli L-serine $\mathrm{O}$-acetyltransferase on the basis of crystal structure: desensitization to feedback inhibition by L-cysteine. Protein Eng Des Sel 19(4):163-167. doi:10.1093/protein/gzj015

Kredich NM (1992) The molecular basis for positive regulation of cys promoters in Salmonella typhimurium and Escherichia coli. Mol Microbiol 6(19):2747-2753

Kredich NM (2008) Biosynthesis of cysteine. EcoSal Plus. doi:10.1128/ ecosalplus.3.6.1.11

Nonaka G, Takumi K (2011) L-cysteine-producing bacterium and a method for producing L-cysteine. US Patent 8,008,048 (B2), 30 Aug 2011

Oguri T, Schneider B, Reitzer L (2012) Cysteine catabolism and cysteine desulfhydrase (CdsH/STM0458) in Salmonella enterica serovar typhimurium. J Bacteriol 194(16):4366-4376. doi:10.1128/jb.00729-12

Ohtsu I, Wiriyathanawudhiwong N, Morigasaki S, Nakatani T, Kadokura $H$, Takagi H (2010) The L-cysteine/L-cystine shuttle system provides reducing equivalents to the periplasm in Escherichia coli. J Biol Chem 285(23):17479-17487. doi:10.1074/jbc.M109.081356

Ohtsu I, Kawano Y, Suzuki M, Morigasaki S, Saiki K, Yamazaki S, Nonaka G, Takagi $H$ (2015) Uptake of L-cystine via an ABC transporter contributes defense of oxidative stress in the L-cystine export-dependent manner in Escherichia coli. PLoS ONE 10(3):e0120619. doi:10.1371/journal.pone.0120619

Sambrook JF, Russell DW (2001) Molecular cloning: a laboratory manual, 3rd edn. Cold Spring Harbor, New York

Shimada T, Tanaka K, Ishihama A (2016) Transcription factor DecR (YbaO) controls detoxification of L-cysteine in Escherichia coli. Microbiology 162(9):1698-1707. doi:10.1099/mic.0.000337

Snel B, Lehmann G, Bork P, Huynen MA (2000) STRING: a web-server to retrieve and display the repeatedly occurring neighbourhood of a gene. Nucleic Acids Res 28(18):3442-3444

Sorensen MA, Pedersen S (1991) Cysteine, even in low concentrations, induces transient amino acid starvation in Escherichia coli. J Bacteriol 173(16):5244-5246

Takumi K, Nonaka G (2016) Bacterial cysteine-inducible cysteine resistance systems. J Bacteriol 198(9):1384-1392. doi:10.1128/jb.01039-15

Takumi K, Ziyatdinov M, Samsonov V, Nonaka G (2017) Fermentative production of cysteine by Pantoea ananatis. Appl Environ Microbiol. doi:10.1128/ aem.02502-16

Tchong SI, Xu H, White RH (2005) L-cysteine desulfidase: an [4Fe-4S] enzyme isolated from Methanocaldococcus jannaschii that catalyzes the breakdown of L-cysteine into pyruvate, ammonia, and sulfide. Biochemistry 44(5):1659-1670. doi:10.1021/bi0484769

Van Dien S, Iwatani S, Usuda Y, Matsui K, Ueda T, Tsuji Y (2010) Method for determining metabolic flux affecting substance production. US Patent 7,809,511 (B2), 5 Oct 2010

Wada M, Takagi H (2006) Metabolic pathways and biotechnological production of L-cysteine. Appl Microbiol Biotechnol 73(1):48-54. doi:10.1007/ s00253-006-0587-z

Wiriyathanawudhiwong N, Ohtsu I, Li ZD, Mori H, Takagi H (2009) The outer membrane TolC is involved in cysteine tolerance and overproduction in Escherichia coli. Appl Microbiol Biotechnol 81(5):903-913. doi:10.1007/ s00253-008-1686-9

Yamada S, Awano N, Inubushi K, Maeda E, Nakamori S, Nishino K, Yamaguchi A, Takagi H (2006) Effect of drug transporter genes on cysteine export and overproduction in Escherichia coli. Appl Environ Microbiol 72(7):4735-4742. doi:10.1128/aem.02507-05

Yamazaki S, Takei K, Nonaka G (2016) ydjN encodes an S-sulfocysteine transporter required by Escherichia coli for growth on S-sulfocysteine as a sulfur source. FEMS Microbiol Lett. doi:10.1093/femsle/fnw185

Zhao C, Kumada Y, Imanaka H, Imamura K, Nakanishi K (2006) Cloning, overexpression, purification, and characterization of $\mathrm{O}$-acetylserine sulfhydrylase-B from Escherichia coli. Protein Expr Purif 47(2):607-613. doi:10.1016/j. pep.2006.01.002 